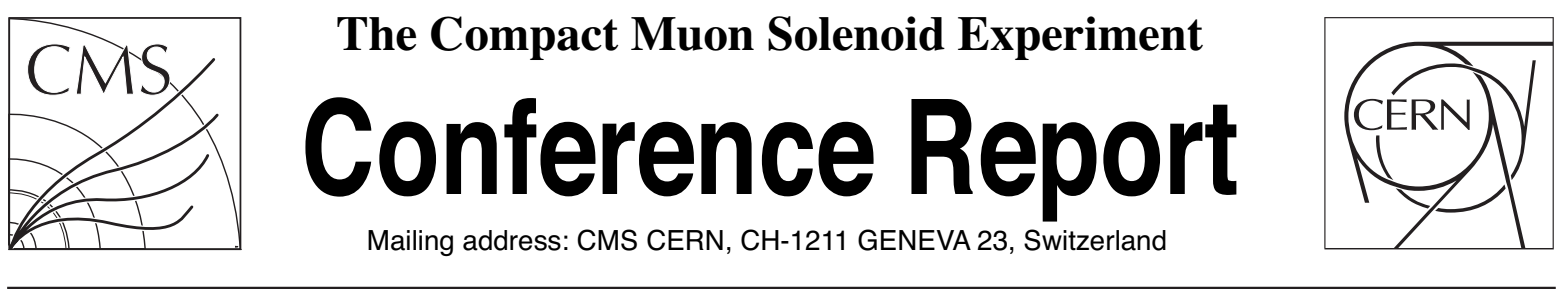

27 November 2008 (v3, 03 December 2008)

\title{
Performances of the CMS Tracker
}

\author{
Chiara Genta on behalf of the CMS Tracker Collaboration
}

\begin{abstract}
With a total area of almost 200 square meters, about 15000 silicon modules, and nearly 10 million readout channels, the CMS Silicon Strip Tracker is by far the largest silicon strip detector ever built. Inside the Strip Tracker, a Pixel Detector made of three barrel layers closed by two forward/backward disks on each side of the interaction region, provides a crucial contribution to pattern recognition, as well as primary and secondary vertices reconstruction. Altogether the Tracker reconstructs the trajectories of charged particles, measures their momentum, and plays a major role in lepton identification and heavy quark tagging. The strip detector has been integrated and commissioned in a dedicated assembly hall on the surface, then inserted in CMS, and re-commissioned using cosmic triggers from the CMS muon system. Excellent results have been achieved in terms of detector performance and preliminary alignment results. The pixel barrel and forward detectors have been built and commissioned separately, and then integrated in CMS. Re-commissioning with the rest of the detector has been done with cosmic triggers.
\end{abstract}

Presented at Crimea 2008- NEW TRENDS IN HIGH-ENERGY PHYSICS,September 27 - October 4, 2008, Yalta,Ukraina,30/11/2008 


\title{
Performances of the CMS Tracker
}

\author{
Chiara Genta \\ on behalf of the CMS Tracker Collaboration
}

\begin{abstract}
With a total area of almost 200 square meters, about 15000 silicon modules, and nearly 10 million readout channels, the CMS Silicon Strip Tracker is by far the largest silicon strip detector ever built. Inside the Strip Tracker, a Pixel Detector made of three barrel layers closed by two forward/backward disks on each side of the interaction region, provides a crucial contribution to pattern recognition, as well as primary and secondary vertices reconstruction. Altogether the Tracker reconstructs the trajectories of charged particles, measures their momentum, and plays a major role in lepton identification and heavy quark tagging. The strip detector has been integrated and commissioned in a dedicated assembly hall on the surface, then inserted in CMS, and re-commissioned using cosmic triggers from the CMS muon system. Excellent results have been achieved in terms of detector performance and preliminary alignment results. The pixel barrel and forward detectors have been built and commissioned separately, and then integrated in CMS. Re-commissioning with the rest of the detector has been done with cosmic triggers.
\end{abstract}

Key words: LHC, CMS, Tracker, Silicon, Micro-strip, Pixel

\section{Introduction}

The Compact Muon Solenoid (CMS)[4] is one of the two general-purpose experiments installed at the Large Hadron Collider (LHC) situated at CERN (Geneva). CMS was designed for a wide range of physics goals, including: the discovery of Higgs boson, elucidation of the electroweak symmetry breaking mechanism, and the search for physics beyond the Standard Model. The accelerator will provide $p p$

Chiara Genta

INFN and University of Florence, via Sansone 1 - I500019 Sesto Fiorentino (FI) - Italy, e-mail: genta@fi.infn.it 


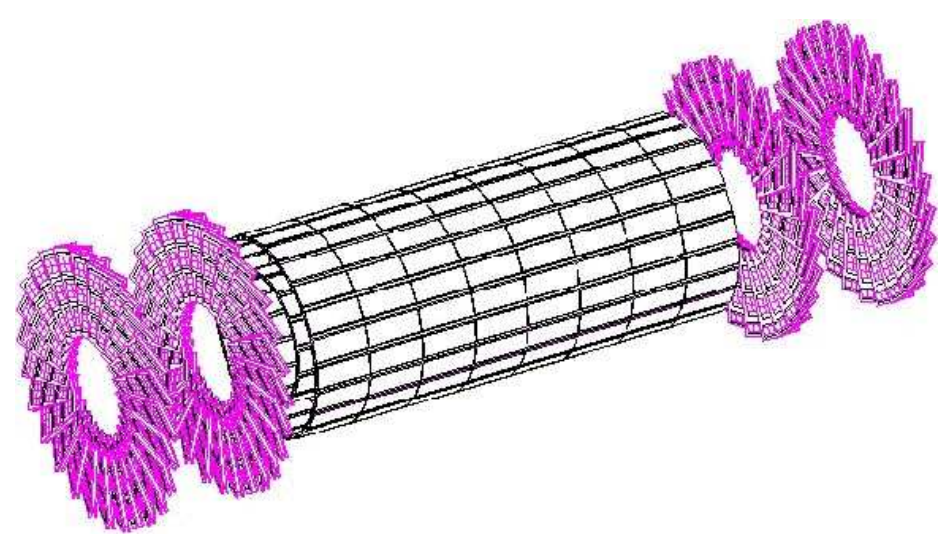

Fig. 1 Three-dimensional view of the silicon pixel detector

collisions at $\sqrt{\mathrm{s}}=14 \mathrm{TeV}$ with a design luminosity of $10^{34} \mathrm{~cm}^{-2} \mathrm{~s}^{-1}$ and a bunch crossing frequency of $40 \mathrm{MHz}$.

Due to the expected high multiplicity of tracks per event, the tracker will play a very important role in the experiment.

\section{The CMS Tracker}

The CMS collaboration opted for a tracker made entirely of silicon modules. In the innermost region a pixel detector is installed, while the outer part is instrumented with $\sim 15000$ silicon micro-strip modules corresponding to a total active area of almost $200 \mathrm{~m}^{2}$. The tracker occupies a volume of $5.4 \mathrm{~m}$ length and $2.4 \mathrm{~m}$ diameter and is immersed in a magnetic field of $\sim 4 \mathrm{~T}$ in order to obtain the design chargedparticle transverse momentum resolution of $1-2 \%$ at $P_{T} \sim 100 \mathrm{GeV} / \mathrm{c}$. The whole tracker covers the pseudorapidity, $\eta$ region between -2.5 and 2.5 .

\subsection{The Silicon Pixel Detector}

The pixel tracker is the detector closer to the beam pipe. Its purpose is to reconstruct with high precision primary and secondary vertices, determine with high accuracy the track impact parameter and to provide a robust seeding for the track patternrecognition. The pixel detector therefore will have a key role in identifying jets from $b$ quarks.

The detector consists of three barrel layers (BPix) with two end-cap disks (FPix) on each side of the barrel as shown in Fig. 1. 

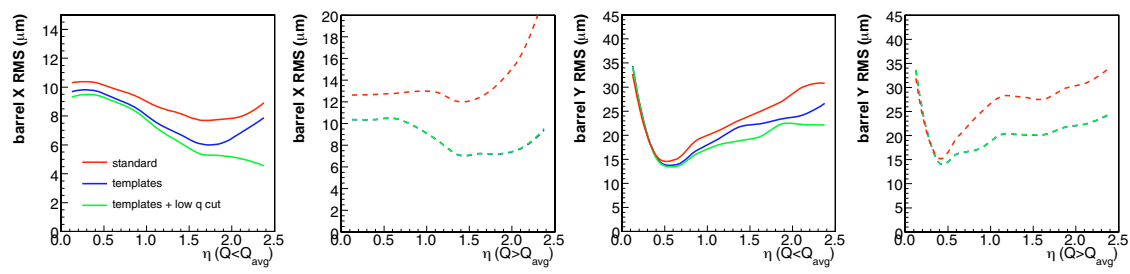

Fig. 2 Pixel $r-\phi$ (labelled as "X") and $z$ (labelled as "Y") resolutions as a function of eta on simulated data. $Q$ is the cluster charge and $Q_{\text {avg }}$ the average cluster charge.

The $53 \mathrm{~cm}$ long BPix layers are located at mean radii of 4.4, 7.3, and $10.2 \mathrm{~cm}$. The FPix disks extending from 6.1 to $15.0 \mathrm{~cm}$ in radius are placed on each side at $z= \pm 34.5 \mathrm{~cm}$ and $z= \pm 46.5 \mathrm{~cm}$. The sensors are $n$-on- $n$ with $n^{+}$implants on $300 \mu \mathrm{m}$ thick $n$ bulk silicon and contain arrays of pixels with a cell size of $100 \times 150 \mu \mathrm{m}^{2}$. The total number of channels is 48 million in the barrel and 18 million in the forward. Sensors are connected to the read out chips (ROC) [9] with indium (barrel) and lead-tin (forward) bump bonding. Each ROC provides an ana$\log$ readout with zero suppression of a $52 \times 80$ pixel-matrix, organized in 26 double columns. The forward detectors are tilted at $20^{\circ}$ in a turbine-like geometry in order to exploit the Lorentz drift in the magnetic field. The effect of the Lorentz angle ( $\sim 25^{\circ}$ on unirradiated sensors) is to spread the charge on more pixel and therefore improve the spatial resolution by charge interpolation. A position resolution of about 10 (15) $\mu \mathrm{m}$ in $r-\phi(\mathrm{z})$ coordinates can be achieved thanks to charge sharing and template reconstruction [11]. In fig. 2 the pixel resolution in $r \phi$ and $z$ coordinates on simulated data is shown.

\subsection{The Silicon Strip Detector}

The Silicon Strip Tracker (SST) consists of four major subsystems, shown in Fig. 3: four layers form the Inner Barrel (TIB) complemented by the Inner Disks (TID) - formed of three disks on each side - cover the $24 \mathrm{~cm}<r<55 \mathrm{~cm}$ and $|z|<120 \mathrm{~cm}$ region; the six layers of the Outer Barrel (TOB) covering approximately $55 \mathrm{~cm}<r<120 \mathrm{~cm}$ and the same $z$ range as the Inner part and $2 \times 9$ disks the two End Caps (TEC), which complete the pseudorapidity coverage up to $|\eta|<2.5$.

The subdetectors are instrumented with microstrip modules in 27 different sizes and shapes. In the barrel modules are rectangular while in the TID and TEC discs are wedge shaped. The strips are $p^{+}$implants on a $n$-type bulk sensors. In the innermost layers (in the barrel) and rings (in the forward) modules are $320 \mu \mathrm{m}$ thick, while in the outer layers are $500 \mu \mathrm{m}$ thick.

In addition, some layers and innermost rings are equipped with special stereo modules, made of sandwiches of sensors with strips tilted by $100 \mathrm{mrad}$ with respect 


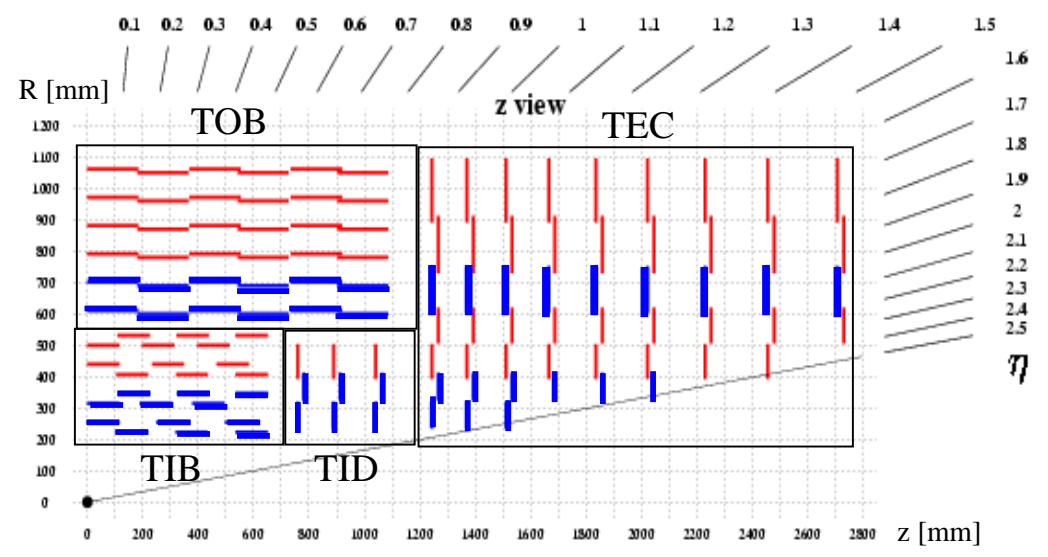

Fig. 3 Layout of one quarter of the CMS silicon strip tracker in the $R-Z$ plane showing the position of the active sensors. Dimensions are in $\mathrm{mm}$. The pseudorapidity coverage is also shown.

to each other, such providing also $z$ information for barrel detectors and $r$ information for disks.

Groups of 128 strips are connected though a pitch adapter to the front-end readout chips (APV25) [7] mounted on the multilayer kapton hybrid circuit. The APV25 is a 128-channel chip built in radiation hard $0.25 \mu \mathrm{m}$ CMOS technology [10]. Each channel consists of a preamplifier coupled to a shaping amplifier which produces a $50 \mathrm{~ns}$ CR-RC pulse shape. The output of each channel is sampled at $40 \mathrm{MHz}$ and sent to a 192 cell deep pipeline. The pipeline depth allows a programmable level 1 trigger latency of up to $4.8 \mu \mathrm{s}$, with 32 locations reserved for buffering events awaiting readout [2].

\subsection{Tracker material budget}

Once the assembly of the SST was complete, it was possible to include in the simulation a realistic description of the material used in the integration.

One of the drawbacks of having such a high number of channels in the tracker is a high material budget due to the subdetector services (power cables, cooling pipes..). As shown in picture 4 the active sensors contribute only for a small amount to the total material. The most critical region is around $1.2<|\eta|<1.8$ where the thickness of the tracker reaches 1.8 radiation lengths $\left(X_{0}\right)$. As can be seen in Fig. 3 this region corresponds to the gap between the barrel and the forward parts of the strip tracker were the service connections are routed. However in the central part of the tracker $(|\eta|<1)$ the material keeps below $0.6 X_{0}$. 

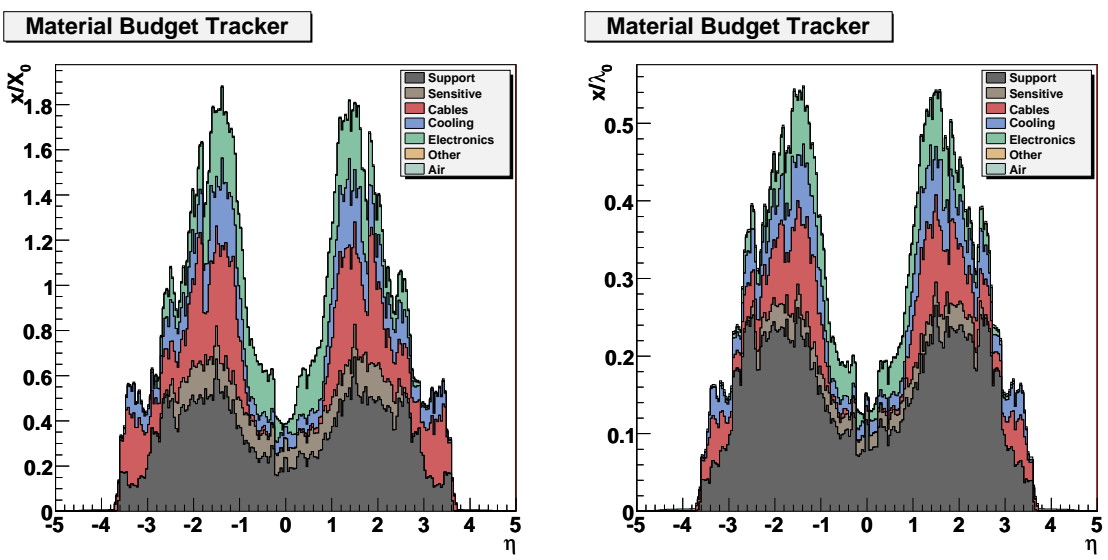

Fig. 4 Material budget in units of radiation lengths (left) and interaction lengths (right) as a function of $\eta$. The contribution of the different materials is also shown.

\section{Tracker Integration Facility}

The modular structure of the tracker made possible to assemble the subdetectors in different institutes all over the world. The construction of the silicon strip tracker was performed during 2006. The final assembly of the Silicon Strip Tracker (STT) in the Support Tube was carried out in March 2007 at CERN in a large, purpose-built, clean area: the Tracker Integration Facility (TIF). After the installation a sector of the SST on the $+z$ side corresponding to the $15 \%$ of the entire detector was cabled and read out, and finally commissioned with cosmic rays in the so called "Sector Test".

For this purpose scintillators put on top and bottom of the tracker were put in coincidence to deliver a cosmic muon as trigger. Between the tracker and the bottom scintillator $5 \mathrm{~cm}$ lead bricks were placed in order to filter very low energy particles. The thickness of the bricks was limited by the clearance below the tracker. During the tests, the scintillators area was gradually increased, allowing to cover larger portions of the tracker, while keeping the $15 \%$ of it readout. In the final configuration the trigger rate was $6.5 \mathrm{~Hz}$, but since the Data Acquisition software (DAQ) was limited to about $3 \mathrm{~Hz}$ by the Front End Driver board (FED) readout [2], a trigger veto was implemented to keep the rate under that level. Five million of events were recorded between March and June 2007 at different temperatures (from $+15{ }^{\circ} \mathrm{C}$ to $-15^{\circ} \mathrm{C}$ ). The test progressed in an incremental way, beginning with testing separately the sub-systems, then proceeding to a test of the barrel systems, and finally incorporating one endcap.

During the Sector Test, practical experience of the operation of the systems (Data Acquisition, Data Quality Monitoring, Control, Safety, Cooling etc.) was achieved. In the following paragraphs some of the results achieved by the analysis of the data will be shown. 


\subsection{Noise Performances}

In the silicon strip modules a linear dependence of the noise from the strip length is expected. In the Sector Test the modules were mounted on the final structure and also other effects like grounding loops, cross talk with other modules, digital noise.., could contribute.

Pedestals and noise were measured on daily basis before any cosmic data taking run (physics run). In order to compare noise from different APVs, a gain correction factor was applied, such that the different responses of the electro-optical readout chain were taken into account. The gain factor was obtained by measuring the height of the APV of the synchronization pulse called tick mark. Since the height of the tick mark depends on the module operating voltages, a systematic spread of $5 \%$ between different layers is expected.

Noise studies permitted to identify noisy strips and bad behaving modules or broken fibers. Modules with known problems were removed either from DAQ or from the data analyses. The resulting fraction of missing modules was at the $0.5 \%$ level. Dead fibers were identified during a synchronization run on the basis of low tick mark height. The number of missing fibers in the Tracker was at the $0.1 \%$ level. The number of dead channels was very constant among several runs for all subdetectors, showing that the identification of these channels is clear and stable: the majority of the dead strips (70\%) were flagged in all runs [5]. The noisy components were instead subject to fluctuations and only a small fraction of the noisy strips were noisy throughout the Sector Test.

\subsection{Signal-to-Noise Performances}

The signal to noise ratio should be largely independent on the different gain corrections, therefore this quantity can be used to compare the performances of the modules in the different layers. Since the signal depends on the path length of the track in silicon, it was normalized to the detector thickness [5]. The noise $N$ was defined as: $N=\sqrt{\sum_{i} N_{i}^{2} / n_{\text {strips }}}$ where $N_{i}$ is the noise of the $i$-th strip of the cluster and $n_{\text {strips }}$ is the number of strips of the cluster. The signal to noise was measured for different cooling temperatures and, as expected, improves with lower temperatures. As can be seen in picture 5 the signal to noise is stable within runs taken at the same temperature.

\subsection{Hit efficiency}

The efficiency of a Tracker module to observe a hit when traversed by a particle, was measured for all modules of a given layer at a time. The pattern recognition 

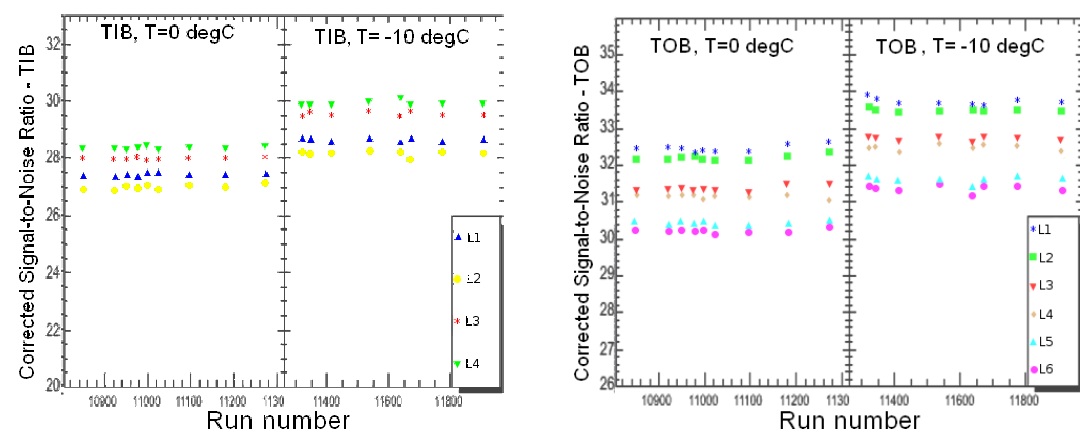

Fig. 5 Signal over noise in the TIB (left) and TOB (right) corrected for the track angle as a function of the run number.

was performed with the Combinatorial Kalman Filter (CKF) [1] excluding the hit on the modules of the layer where the efficiency is calculated. A sample of high quality events was selected by requiring only one track reconstructed by the CKF algorithm, one hit in the first TIB layer, one hit in each of the two outermost TOB layers, and at least four reconstructed hits of which at least three in double sided layers. The efficiency for a module was measured by asking for an intersection with the interpolated track and by checking for the presence of a hit. For increased robustness in the presence of residual misalignment the distance between the hit and the predicted track position was not used in the selection. An upper cut of $30^{\circ}$ on the angle of incidence of the track with respect to the normal to the module plane, applied in TIB layer 2, selected topologies similar to the ones expected from collisions. In order to avoid artificial inefficiencies at the edge of the sensitive region a fiducial area was used to restrict the region in which efficiency is measured. The hit reconstruction efficiency exceeds $99.8 \%$ for all measured layers, as it is shown in fig. 6.
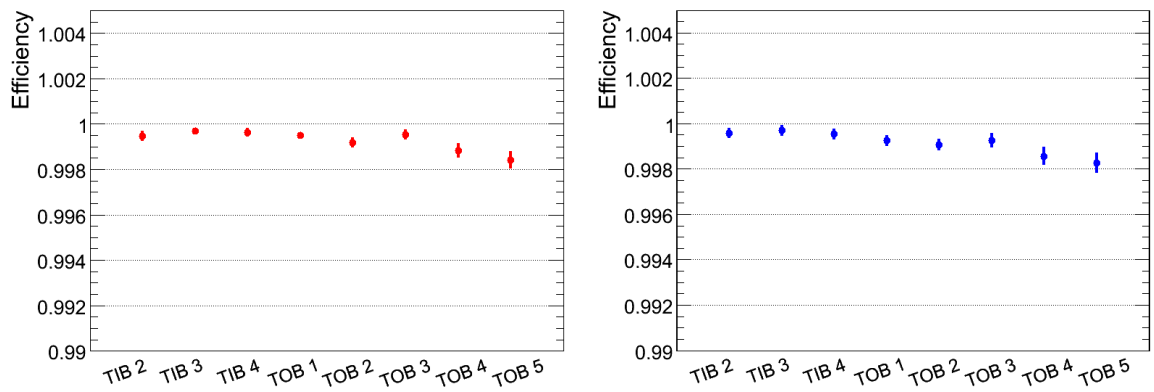

Fig. 6 Summary of the layer efficiency at room temperature (left) and $-10^{\circ} \mathrm{C}$ (right). 


\subsection{Track efficiency}

Due to the absence of an external reference other than the scintillators used for triggering it was not possible to measure the absolute efficiency of the tracker. Therefore tracks in TIB and TOB were reconstructed independently and two efficiencies were computed: $\varepsilon(T O B \mid T I B)$, the probability to find a matching TOB track for a given TIB track, and, vice versa, $\varepsilon(T I B \mid T O B)$. The track used as reference was required to have at least 2 hits in double sided modules and its extrapolation to be fully contained in the other subdetector. The match between tracks was based on a comparison of the azimuthal angles. The difference was required to be smaller than five times the resolution determined from simulation. Obtained results are in the range 90\%-99\% (Table 1) for the three track reconstruction algorithms used in the Sector Test [1]; the small discrepancies observed with respect to Monte Carlo are due to a different acceptance between reconstructed and simulated data.

Table 1 Average conditional track efficiencies and corresponding statistical uncertainties for all three track reconstruction algorithms in data and Monte Carlo simulation.

\begin{tabular}{|c|c|c|c|c|}
\hline & \multicolumn{2}{|c|}{$\varepsilon(\mathrm{TIB} \mid \mathrm{TOB})[\%]$} & \multicolumn{2}{|c|}{$\varepsilon(\mathrm{TOB} \mid \mathrm{TIB})[\%]$} \\
\hline & Data & MC & Data & $\mathrm{MC}$ \\
\hline Combinatorial Kalman Filter & $\overline{94.0 \pm 0.2}$ & $98.66 \pm 0.04$ & $97.7 \pm 0.1$ & $98.76 \pm 0.04$ \\
\hline Cosmic Track Finder & $93.1 \pm 0.2$ & $94.46 \pm 0.09$ & $96.9 \pm 0.1$ & $97.36 \pm 0.06$ \\
\hline Road Search & $89.9 \pm 0.2$ & $89.08 \pm 0.12$ & $99.0 \pm 0.1$ & $99.39 \pm 0.03$ \\
\hline
\end{tabular}

\subsection{Alignment}

Alignment analysis with tracks is based on the minimization of the track-hit residuals. More precisely, one can minimize the $\chi^{2}$ function which includes the covariance matrix $V$ of the measurement uncertainties:

$$
\chi^{2}=\sum_{i}^{\text {tracks }} r_{i}^{T}(p, q) V_{1}^{-1} r_{i}(p, q)
$$

where $p$ represents the position and orientation of the modules and $q$ the track parameters.

At the sector test three different track based alignment methods were employed [6]:

- Hits and Impact Points (HIP): an iterative procedure to find a local analytical solution for $p$ only [8];

- Kalman filter fit method: a sequential procedure updating alignment parameters after adding every track [12]; 
- MillePede minimization: a method to find a global solution for $p$ and $q$, taking into account all possible correlations [3].

Due to the absence of the magnetic field during the sector test it was not possible to estimate the track momentum. For this reason and the low momentum spectrum of the cosmic muons, the alignment accuracy was limited by the uncertainty on the multiple scattering. Moreover due to the angular distribution of the cosmic rays the alignment of the endcaps was very difficult and it was only possible to align the TEC at the disk level. The typical achieved precision on module position measurement in the local $x$ coordinate is estimated to be about $50 \mu \mathrm{m}$ and $80 \mu \mathrm{m}$ in the Tracker Outer and Inner Barrels, respectively.

\section{The Global Run}

In December 2007 the SST was installed underground in CMS and the full cabling and piping was completed in March 2008. Almost all the Silicon Strip Tracker (TIB, TID, TOB and TEC+) participated to the CMS global run starting from July 2008. By the end of July also the installation of the pixel detector was completed and starting from 22 August all the tracker was included in the global data acquisition. The re-commissioning of all the tracker is now completed, and the tracker performances are excellent. A huge amount of cosmic data has been taken both with and without magnetic field. In fig. 7 a cosmic event recorded during a run with magnetic field on is shown.

The analysis of the data is ongoing. The data with magnetic field will permit to calibrate the Lorentz angle and will improve the track reconstruction and the alignment.

\section{Conclusions}

The complete commissioning of the tracker and the analysis of the sector test data has demonstrated the good quality of the pixel and strip modules. The data taken since August 2008 until November 2008 during the global run will improve the understanding of the detector and will provide the first calibration constants that will be used with collision data.

\section{References}

1. W. Adam et al., Track Reconstruction with Cosmic Ray Data at the Tracker Integration Facility, CMS NOTE in preparation. 


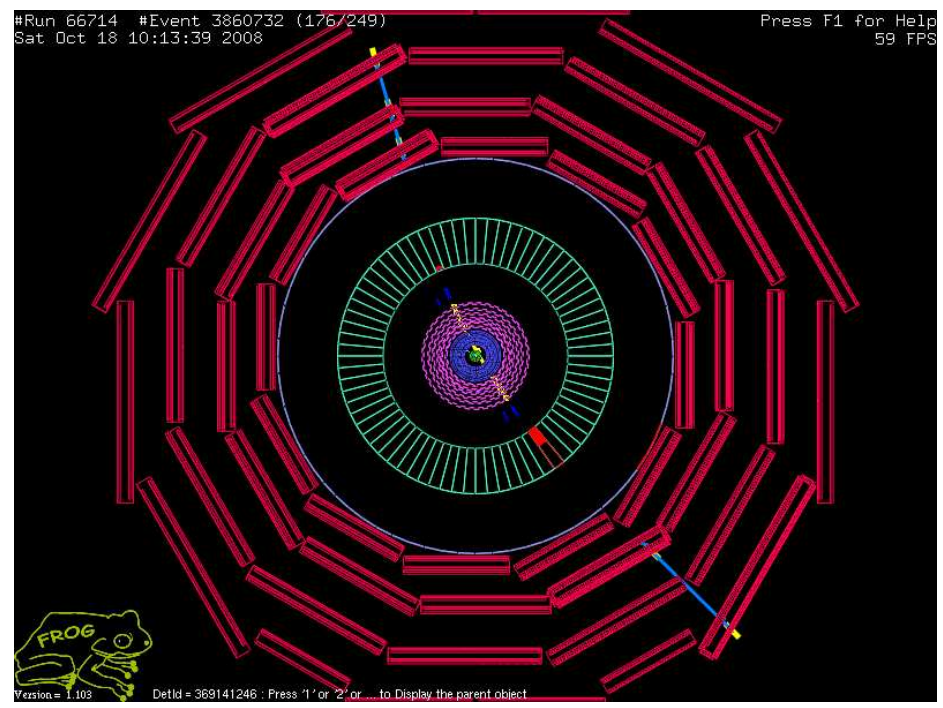

Fig. 7 Cosmic track crossing the CMS detector including all the tracker layers, shown in the innermost parts of the picture.

2. S.A. Baird et al., The Front-End Driver card for the CMS Silicon Strip Tracker Readout, Eighth Workshop on Electronics for LHC Experiments, CERN/LHCC/2002-034.

3. V. Blobel, Software Alignment for Tracking Detectors, Nucl. Instrum. Meth. A566 (2006) 5.

4. M.Della Negra et al., CMS Physics Technical Design Report: Volume 1, Detector Performance and Software, CERN LHCC-2006-001, 2006

5. L. Demaria et al., Silicon Strip Tracker Detector Performance with Cosmic Ray Data at the Tracker Integration Facility, CMS NOTE-2008/032.

6. G. Flucke et al., CMS Tracker Alignment at the Integration Facility, CMS NOTE in preparation.

7. M. J. French et al., "Design and results from the APV25, a deep sub-micron CMOS front-end chip for the CMS tracker," Nucl. Instrum. Meth., vol. A466, pp. 359-365, 2001.

8. V. Karimaki, T. Lampen, F.-P. Schilling, The HIP Algorithm for Track Based Alignment and its Application to the CMS Pixel Detector, CMS NOTE-2006/018.

9. H. C. Kastli et al., Design and performance of the CMS pixel detector readout chip, Nucl.Instrum.Meth.A565:188-194,2006.

10. A. Marchioro, Deep submicron technologies for HEP, Proceedings of 4th workshop on electronics for LHC experiments, CERN/LHCC/98-36,40-46.

11. M. Swartz, D. Fehling, G. Giurgiu, P. Maksimovic, V. Chiochia, A new technique for the reconstruction, validation, and simulation of hits in the CMS Pixel Detector, CMS NOTE2007/033

12. E. Widl, R. Frühwirth, W. Adam, A Kalman Filter for Track-based Alignment, CMS NOTE$2006 / 022$ 